\title{
SINGULARITIES AND GROUP ACTIONS
}

\author{
BY PETER ORLIK ${ }^{1}$ \\ To my parents
}

1. Introduction. The emergence of Transformation Groups as a separate branch of mathematics about a hundred years ago is connected with the names of Sophus Lie and Felix Klein. The invariant theorists of the day asked to find all invariant polynomials of a given linear group $G$. Let us fix the field $\mathbf{C}$ and let $f: \mathbf{C}^{n+1} \rightarrow \mathbf{C}$ be a polynomial mapping. A related question asks for $\operatorname{Aut}(f)$, the group of linear transformations leaving $f$ fixed. Call $\mathbf{z}$ a critical point of $f$ if the partial derivatives $\partial f / \partial z_{i}$ vanish at $\mathbf{z}$. A critical point is called isolated if it is the only critical point in some neighborhood of $\mathbf{z}$. In the context of invariant theory $f$ is homogeneous of degree $m$. If $m \geqslant 2$, then 0 is a critical point, and if it is isolated, then it is the only critical point of $f$. Lie [49] noted (without proof; for history and a proof see Orlik and Solomon [59]):

THEOREM. If $G$ is a linear group leaving a homogeneous polynomial $f$ of degree $m \geqslant 3$ invariant, and the critical point of $f$ at 0 is isolated, then $G$ is finite. In particular, $\operatorname{Aut}(f)$ is finite.

In his famous lectures on the icosahedron Klein [45] observed the connection between the binary icosahedral group $G$ and the polynomial $f\left(z_{0}, z_{1}, z_{2}\right)$ $=z_{0}^{5}+z_{1}^{3}+z_{2}^{2}$. The ring of invariant polynomials of a representation of $G$ in $S U(2)$ is generated by three homogeneous polynomials of degrees $6,10,15$. There is one polynomial dependence among them which (up to coefficients) reads $z_{0}^{5}+z_{1}^{3}+z_{2}^{2}=0$. Thus $\mathbf{C}^{2} / G$ is isomorphic to the hypersurface $V=$ $f^{-1}(0)$ in $\mathbf{C}^{3}$. In fact $V$ is the cone over Poincaré's dodecahedral space. The critical point 0 of $f$ is isolated and it is also called an isolated singularity of $V$. Such a singular point has a (nonunique) resolution, consisting of a nonsingular algebraic variety $\tilde{V}$ and a proper map $\pi: \tilde{V} \rightarrow V$ such that the restriction $\pi$ : $\tilde{V}-\pi^{-1}(\mathbf{0}) \rightarrow V-\mathbf{0}$ is an isomorphism. Finding a resolution is in general rather cumbersome. However, this polynomial has a symmetry which may be exploited, in that for $t \in C$ we have $f\left(t^{6} z_{0}, t^{10} z_{1}, t^{15} z_{2}\right)=t^{30} f\left(z_{0}, z_{1}, z_{2}\right)$, so $V$ is invariant under the action of the multiplicative group of nonzero complex numbers, $\mathbf{C}^{*}$. We shall return to this in $\$ 7$.

This survey will consider some highlights of the interaction between Transformation Groups and Singularities from the last decade. I wish to thank I. Dolgachev, A. Durfee, H. Hamm, L. Kauffman, W. Neumann, T. Petrie, R.

An invited address delivered at the 762nd meeting of the Society at Chicago, Illinois, November 12, 1978; received by the editors January 25, 1979.

AMS (MOS) subject classifications (1970). Primary 57E25; Secondary 57D45, 14 D05.

1 Partially supported by NSF. 
Randell, R. Schultz and P. Wagreich for providing valuable suggestions and references.

2. Degree of symmetry. In the case of a homogeneous polynomial $f$, the group $\operatorname{Aut}(f)$ is a measure of its symmetry. Given an arbitrary compact, connected smooth manifold $\boldsymbol{M}^{\boldsymbol{m}}$, one possible generalization is to consider the dimension of the largest compact Lie subgroup $G$ of $\operatorname{Diff}\left(M^{m}\right)$. This is called the degree of symmetry, $N\left(M^{m}\right)$ by W. Y. Hsiang [41]. It is a rather coarse invariant, which nevertheless has interesting properties. Since $M$ admits a $G$-invariant Riemannian metric, the elements of $G$ operate as isometries of $M$ and the methods of differential geometry may be applied. In fact the following was known already fifty years ago, see Eisenhart [26, p. 239].

ThEOREM. For all $M^{m}, N\left(M^{m}\right) \leqslant \frac{1}{2} m(m+1)$ and $N\left(M^{m}\right)=\frac{1}{2} m(m+1)$ if and only if $M^{m}$ is the (standard) $m$-sphere $S^{m}$ or projective space $P^{m}$.

It was not envisioned then that the topological sphere could support exotic smooth structures. The degree of symmetry of an exotic sphere is considerably less than that of the standard sphere. For example Hsiang [41] proved that if $K$ is an exotic $(4 k+1)$-sphere, then $N\left(K^{4 k+1}\right) \leqslant k(2 k+1)+1$. One may ask which are the "most symmetric" exotic spheres. These were found when Brieskorn [9] discovered that exotic spheres "occur in nature," i.e. that for certain polynomials $f: \mathbf{C}^{n+1} \rightarrow \mathbf{C}$ with an isolated critical point at $\mathbf{0}$, we may choose a small sphere $S_{\varepsilon}^{2 n+1}$ centered at 0 so that the link $K=S_{\varepsilon}^{2 n+1} \cap$ $f^{-1}(0)$ is an exotic sphere.

Consider the polynomial given by $f(z)=z_{0}^{3}+z_{1}^{2}+\cdots+z_{2 k+1}^{2}$. If $\operatorname{SO}(2 k$ $+1)$ acts on the coordinates $\left(z_{1}, \ldots, z_{2 k+1}\right)$ as a subgroup of $U(2 k+1)$ then $f$ is invariant. Moreover, $f^{-1}(0)$ is invariant under the $S^{1}=U(1)$ action given by

$$
t\left(z_{0}, z_{1}, \ldots, z_{2 k+1}\right)=\left(t^{2} z_{0}, t^{3} z_{1}, \ldots, t^{3} z_{2 k+1}\right)
$$

Thus the link $K^{4 k+1}$ has $N(K) \geqslant k(2 k+1)+1$. It turns out that for $k \neq 2^{j}$ $-1 K$ is an exotic $(4 k+1)$-sphere, so $N\left(K^{4 k+1}\right)=k(2 k+1)+1$. Since $N\left(S^{4 k+1}\right)=(4 k+1)(2 k+1)$, we may say with tongue in cheek that the standard sphere is roughly four times more symmetric than the exotic sphere $K^{4 k+1}$. For sharper limits on the degree of symmetry of other exotic spheres, see Schultz [81].

3. Exotic actions. Let $S^{2 n+1}$ be the standard sphere and $T: S^{2 n+1} \rightarrow S^{2 n+1}$ a smooth free involution, i.e. $T(x) \neq x$ for all $x$, and $T^{2}=\mathrm{id}$. Then $Q^{2 n+1}=$ $S^{2 n+1} / T$ is a smooth manifold homotopy equivalent to projective space, $P^{2 n+1}$. We call $T$ an exotic involution if $Q$ is not diffeomorphic to $P$. Exotic involutions were first found by Hirsch and Milnor [39] and Bredon [8]. A particular class is easily described using the Brieskorn polynomials $f(\mathbf{x})=z_{0}^{d}$ $+z_{1}^{2}+\cdots+z_{2 k+1}^{2}, k \geqslant 1, d$ odd. It may be shown that the link $K(d)$ of the singularity at 0 is a standard sphere if $d \equiv \pm 1 \bmod 8$. The involution 
$T\left(z_{0}, z_{1}, \ldots, z_{2 k+1}\right)=\left(z_{0},-z_{1}, \ldots,-z_{2 k+1}\right)$ is free on $K$. Atiyah and Bott [6] use their fixed point formula to show that depending on $d$ there are $2^{2 k}$ distinct exotic involutions among these, see also Giffen [30].

Another interesting question is whether a given group can act on a given manifold or class of manifolds. For example linear free actions of finite groups on spheres have been classified. A necessary condition on the group for the existence of such an action is that all subgroups of order $p q$ be cyclic ( $p, q$ are not necessarily distinct primes). It is therefore natural to ask whether the meta-cyclic group of order $p q$ can act freely on any homotopy sphere. Petrie [66] gives a construction whose starting point is one of the singularities in consideration. Let $\pi=\left\{x, y \mid x^{p}=y^{q}=1, y x y^{-1}=x^{\sigma}\right\}, p$ odd, $q$ odd prime, $\sigma$ a primitive $q$ th root of $1 \bmod p$ and $(\sigma-1, p)=1$. Let $\pi_{p}$ be the normal subgroup generated by $x$ and $\pi_{q}$ the subgroup generated by $y$, which is also the quotient group $\pi / \pi_{p}$. Thus $1 \rightarrow \pi_{p} \stackrel{i}{\rightarrow} \pi \stackrel{j}{\rightarrow} \pi_{q} \rightarrow 1$. Let $V_{p}$ and $V_{q}$ be one-dimensional complex representations of $\pi_{p}$ and $\pi_{q}$. Let $\mathbf{C}[\pi]$ resp. $\mathbf{C}\left[\pi_{p}\right]$ be the group rings of $\pi$ and $\pi_{p}$. Let $i_{*} V_{p}=\mathrm{C}[\pi] \otimes_{\left.\mathrm{C}_{\pi_{p}}\right]} V_{p}$ and let $j^{*} V_{q}$ be the one-dimensional complex representation of $\pi$ defined by $V_{q}$ viewed as a $\mathrm{C}[\pi]$ module via $j$. Then $V=i_{*} V_{p} \oplus j^{*} V_{q}$ is a complex $(q+1)$-dimensional representation of $\pi$. If $z_{1}, \ldots, z_{q}$ are complex coordinates for $i_{*} V_{p}$ and $z_{q+1}$ is a complex coordinate for $j^{*} V_{q}$, then the polynomial

$$
f\left(z_{1}, \ldots, z_{q+1}\right)=z_{1}^{p}+z_{2}^{p}+\cdots+z_{q}^{p}+z_{q+1}^{l}
$$

with $l=q^{n}$ is invariant under the action of $\pi$. With proper choice of $t$ and $\varepsilon$, $\pi$ acts freely on $K_{t}=f^{-1}(t) \cap S_{e}$. Unfortunately, $K_{t}$ is not a homotopy sphere. It may be altered to yield the desired result by equivariant surgery, see Petrie [66] for the nontrivial details.

4. Milnor fibration. Let $f: \mathbf{C}^{n+1} \rightarrow \mathbf{C}$ be a polynomial map with a critical point at 0 . Let $S_{\varepsilon}^{2 n+1}$ be a small sphere about 0 , let $V_{t}=\left\{z \in \mathbf{C}^{n+1} \mid f(z)=t\right\}$ and $K_{t}=S_{e}^{2 n+1} \cap V_{t}$. Milnor [51] proves the following.

THEOREM. The mapping $f /|f|: S^{2 n+1} \backslash K_{0} \rightarrow S^{1}$ is the projection of a smooth fiber bundle.

If 0 is an isolated singularity of $V_{0}$, then the following is true:

(i) The typical fiber $F=F_{t}$ is diffeomorphic to $V_{t} \cap B_{e}$ and it is an $(n-1)$-connected parallelizable smooth $2 n$ manifold. The homotopy type of $F$ is that of a bouquet of $n$-spheres whose number, $\mu$ is called the Milnor number of the singularity.

(ii) For $|t| \leqslant \delta, K=K_{t}$ are all diffeomorphic smooth $(n-2)$-connected $(2 n-1)$-manifolds. Moreover, the closure of $F$ is a compact manifold with boundary $K$.

(iii) $S^{2 n+1}-K$ is obtained from $F \times[0,2 \pi]$ via a diffeomorphism $h$ : $F \rightarrow F$ called the characteristic map. The induced map $h_{*}: H_{n}(F) \rightarrow H_{n}(F)$ is called the monodromy.

Thus $S^{2 n+1}$ is decomposed as a union of the codimension 1 submanifolds $F_{t}, t \in[0,2 \pi]$ together with $K_{0}$ in such a way that the closure of each fiber $F_{t}$ 
is $K_{0}$. Such a decomposition is called a book structure or an Alexander decomposition, see Figure 1 below.

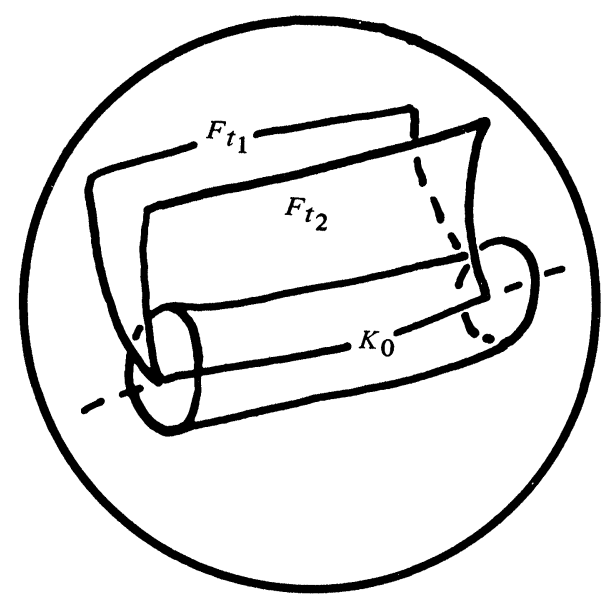

FigURE 1

(iv) For $n \geqslant 3, K$ is simply connected and we have a short exact sequence

$$
0 \rightarrow H_{n}(K) \rightarrow H_{n}(F) \stackrel{I^{*}-h_{*}}{\rightarrow} H_{n}(F) \rightarrow H_{n-1}(K) \rightarrow 0
$$

where $I$ is the identity map. In particular, $H_{n}(K)=H_{n-1}(K)=0$ if and only if $I_{*}-h_{*}$ is an isomorphism. In this case $K$ has the homotopy type of $S^{2 n-1}$ and it is called a homotopy sphere. A theorem of Smale implies that $K$ is in fact homeomorphic to $S^{2 n-1}$. In general $K$ is not diffeomorphic to $S^{2 n-1}$, as we noted in the examples of Brieskorn.

These results have been extended to singularities of complete intersections by Hamm [35].

5. Foliations. Polynomials with singularities played an essential role in the construction of codimension one foliations of all odd dimensional spheres. By a codimension one $C^{\infty}$ foliation of an $m$-manifold $M$ we mean a decomposition of $M$ into a union of disjoint connected codimension one subsets $\left\{\mathcal{L}_{\alpha}\right\}_{\alpha \in A}$ called the leaves, such that every point $x \in M$ has a neighborhood $U$ and a system of local $C^{\infty}$ coordinates $x=\left(x_{1}, \ldots, x_{m}\right): U \rightarrow \mathbf{R}^{m}$ such that for each leaf $\mathfrak{L}_{\alpha}$, the components of $U \cap \mathfrak{L}_{\alpha}$ are described by the equation $x_{m}=0$. If $M$ has boundary, then we require that each component of the boundary be a leaf and the foliation extend to a smooth collaring of $M$ by defining the leaves in the collar $\partial M \times[0,1]$ to be the components of $\partial M \times\{t\}$.

It is easily seen that for compact manifolds the vanishing of the euler characteristic is a necessary condition for a codimension one foliation. This rules out even dimensional spheres. Clearly, $S^{1}$ is foliated by its points. Reeb constructed a foliation on $S^{3}$ as follows. First foliate a strip of the plane by congruent curves, each asymptotic to the boundary as pictured in Figure 2. 


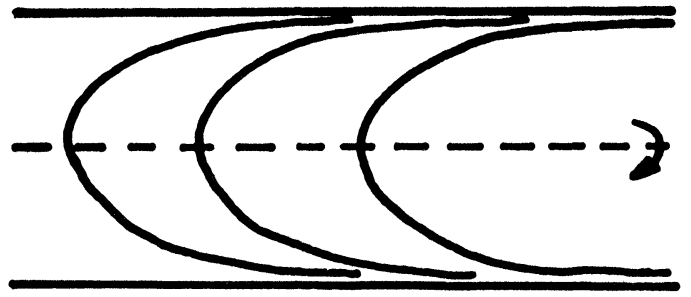

FIGURE 2

Rotate about the center line to obtain a foliation of $R \times D^{2}$. Now divide by $\mathrm{Z}$ using the periodicity of the foliation to obtain a foliation of $S^{1} \times D^{2}$ whose leaves are planes except for the boundary torus. Two such solid tori foliate $S^{3}$. It was natural to ask whether all odd dimensional spheres admit a codimension one foliation.

Notice that if $p: M^{m} \rightarrow N^{n}$ is a smooth fiber bundle and there is a codimension one foliation of $N^{n}$, then $p^{-1}$ pulls it back to a codimension one foliation of $M^{m}$.

We noted that the Milnor fibration decomposes $S^{2 n+1}$ as a disjoint union of the codimension one submanifolds $F_{t}$ together with the link $K$. It is not hard to see that $K$ is embedded with a trivial normal bundle so $K \times D^{2} \subset$ $S^{2 n+1}$. The construction by Lawson [48] and Durfee [22] depends on the following observation. If there is a codimension one foliation of $K \times D^{2}$ such that $\partial\left(K \times D^{2}\right)=K \times S^{1}$ is a leaf, then $S^{2 n+1}$ has a codimension one foliation by "wrapping the leaves around" $K \times S^{1}$. This latter is shown in Figure 3. It remains to obtain a foliation of $K \times D^{2}$. Here they use induction. If $K \times D^{2}$ may be fibered over a lower dimensional sphere which has a codimension one foliation, then the bundle projection pulls the foliation back to the desired foliation of $K \times D^{2}$. Thus it suffices to find polynomials with isolated singularity whose link manifold $K$ fibers over an odd dimensional sphere. Explicit computations show that

$$
f(\mathbf{z})=z_{0}^{2}+z_{1}^{2}+\cdots+z_{n}^{2}, \quad \text { for } n \text { odd }
$$

and

$$
f(\mathbf{z})=\left(z_{0}+z_{1}^{2}\right)\left(z_{0}^{2}+z_{1}^{5}\right)+z_{2}^{2}+\cdots+z_{n}^{2} \text {, for } n \text { even }
$$

have this property. Subsequently Thurston [87] proved that the euler characteristic is the only obstruction to the existence of a codimension one $C^{\infty}$ foliation.
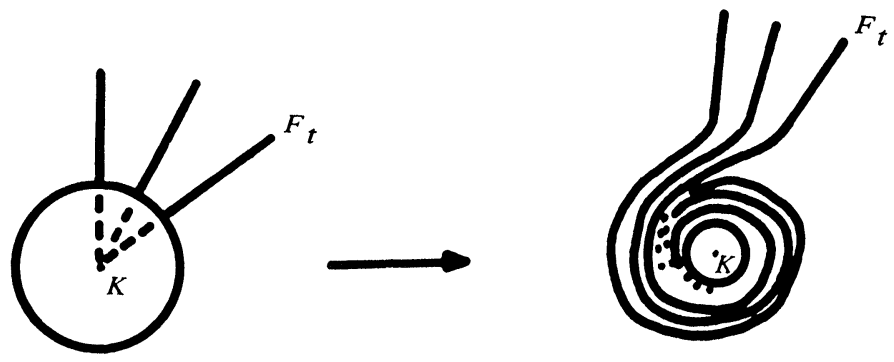

Figure 3 
6. $\mathbf{C}^{*}$-actions. Klein's and Brieskorn's polynomials are examples of a very tractable class called weighted homogeneous or quasihomogeneous polynomials. Call $f\left(z_{0}, \ldots, z_{n}\right)$ quasihomogeneous with degree $d$ and exponents $q_{0}, \ldots, q_{n}$ if $f\left(t^{a_{0} z_{0}}, \ldots, t^{q_{n}} z_{n}\right)=t^{d} f\left(z_{0}, \ldots, z_{n}\right)$. The variety $V=f^{-1}(0)$ admits the corresponding $\mathrm{C}^{*}$-action. We shall assume here that the integers $d ; q_{0}, \ldots, q_{n}$ are all positive (in which case the action is called "good"), but there are situations where it is essential not to make this assumption, see Orlik and Wagreich [63]. In the terminology of Milnor [51] $f$ has weights $w_{i}=d / q_{i}$.

We have noted that one important invariant of an isolated critical point is its Milnor number $\mu$. This number is hard to compute in general, but for quasihomogeneous polynomials we have a result of Milnor and Orlik [53]:

THEOREM. If $f$ is a quasihomogeneous polynomial of type $d ; q_{0}, \ldots, q_{n}$ with an isolated critical point, then

$$
\mu=\prod_{i=0}^{n}\left(d-q_{i}\right) / \prod_{i=0}^{n} q_{i}=\prod_{i=0}^{n}\left(w_{i}-1\right) .
$$

Note that a priori this product is not an integer. This result has been extended to complete intersections by Randell [77] and Hamm and Greuel [37].

Additional information about the Milnor fibration is in the characteristic map $h: F \rightarrow F$ and its induced map in homology, the monodromy. Let $\xi=\exp (2 \pi i / d)$. Then we have $h\left(z_{0}, \ldots, z_{n}\right)=\left(\xi^{q_{0}} z_{0}, \ldots, \xi^{q_{n}} z_{n}\right)$. The eigenvalues of the monodromy transformation $h_{*}$ are computed by Milnor and Orlik [53], but this suffices only to determine the rank of $H_{n-1} K$ and $H_{n-2} K$. In order to find the torsion coefficients of $\mathrm{H}_{n-2} \mathrm{~K}$ it is necessary to obtain the integer matrix of $h_{*}$ with respect to some basis. This been done for some types of quasihomogeneous polynomials by Randell [74] and Orlik and Randell [58], but for arbitrary quasihomogeneous polynomials the problem is still open.

The space $V_{0}-\{0\}$ is invariant under the $\mathbf{C}^{*}$-action and the orbit space $X=V_{0}-\{0\} / \mathbf{C}^{*}$ is in a natural way a compact complex space. The orbit map $\pi: V_{0}-\{\boldsymbol{0}\} \rightarrow X$ is almost a bundle projection with fibers $\mathbf{C}^{*}$. It is called a Siefert bundle. It is interesting to determine when $X$ is a manifold, see Neumann [54], Edmunds [25] and Randell [76]. Many of the algebraic topological and algebraic geometrical invariants of $X$ may be computed explicitly in terms of the integers $d ; q_{0}, \ldots, q_{n}$; see Randell [75], [76].

When $f$ is a sum of polynomials in distinct variables, we have the following result of Oka [56].

THEOREM. Let $f$ be a polynomial in $\mathbf{C}^{n} \times \mathbf{C}^{m}$ such that $f(z, w)=g(z)+h(w)$ for each $(z, w) \in \mathbf{C}^{n} \times \mathbf{C}^{m}$, where $g(z)$ and $h(w)$ are quasihomogeneous polynomials in $\mathbf{C}^{n}$ and $\mathbf{C}^{m}$ respectively. Let $F_{f}=f^{-1}(1) \subset \mathbf{C}^{n} \times \mathbf{C}^{m}, F_{g}=g^{-1}(1)$ $\subset \mathbf{C}^{n}$ and $F_{h}=h^{-1}(1) \subset \mathbf{C}^{m}$. Then there is a natural homotopy equivalence between $F_{f}$ and $F_{g} * F_{h}$, where $F_{g} * F_{h}$ is the join of $F_{g}$ and $F_{h}$ with the strong topology.

Corollary (Sebastiani-Thom [82]). The monodromy of $f$ is the tensor product of the monodromies of $g$ and $h$. 
A particularly simple example is provided by the one-variable case $f(z)=$ $z^{a}$. The fiber $F=f^{-1}(1)$ consists of $a$ points and $\mu=\operatorname{rank} \tilde{H}_{0}(F)={ }^{\prime} a-1$. Choosing a suitable basis the monodromy matrix is given by the companion matrix of the polynomial $\left(t^{a}-1\right) /(t-1)$ :

$$
M_{a}=\left[\begin{array}{ccccc}
0 & 1 & 0 & \ldots & 0 \\
0 & 0 & 1 & \cdots & 0 \\
0 & \cdots & & 0 & 1 \\
-1 & -1 & \ldots & & -1
\end{array}\right]
$$

Thus for the Pham-Brieskorn polynomial

$$
f(z)=z_{0}^{a_{0}}+z_{1}^{a_{1}}+\cdots+z_{n}^{a_{n}}
$$

the monodromy matrix is the tensor product

$$
M=M_{a_{0}} \otimes M_{a_{1}} \otimes \cdots \otimes M_{a_{n}}
$$

The link of $f(z)+w^{p}$ may be viewed as a " $p$-cyclic suspension" of the link of $f(z)$ and there is a periodicity in the signature of the Milnor fiber as a function of $p$. This in turn has connections with knot cobordism groups and the so called knot- and link manifolds, see Jänich [42], Hirzebruch [40], Erle [27], Bredon [8], Durfee and Kauffman [24] and Kauffman and Neumann [43].

Let $f$ be a homogeneous polynomial of degree $m$ with an isolated singularity. Let $G=\operatorname{Aut}(f)$ be the group of linear automorphisms of $f$. If $m=2$, then $f$ is invariant under some orthogonal group e.g. as in \$2. If $m \geqslant 3$ then Lie's theorem quoted in the introduction shows that $G$ is finite. Since $G$ acts on the Milnor fiber, we have a representation of $G$ on $H_{n}(F ; \mathrm{C})$. The character and eigenvalues of this action were computed by Orlik and Solomon [59].

7. Resolution of singularities. Suppose $V$ is an algebraic surface in $\mathbf{C}^{n+1}$ with one singular point $\mathrm{0} \in V$. Then it has a resolution consisting of a nonsingular algebraic surface $\tilde{V}$ and a proper map $\pi: \tilde{V} \rightarrow V$ such that the restriction $\pi: \tilde{V}-\pi^{-1}(\mathbf{0}) \rightarrow V-0$ is an isomorphism. Let $X=\pi^{-1}(0)$. Then $X=\cup{ }_{i=1}^{r} X_{i}$ where each $X_{i}$ is a compact, nonsingular curve and $X_{i} \cap X_{j}$ is either empty or consists of a single normal crossing. Thus $V$ arises by collapsing $X$ to a point in $\tilde{V}$. In particular the link $K$ of $0 \in V$ is diffeomorphic to the boundary of a tubular neighborhood of $X$ in $\tilde{V}$. We may associate a weighted graph $\Gamma$ to the resolution as follows. To each curve $X_{i}$ assign a vertex $e_{i}$ weighted by a nonnegative integer $g_{i}$, the genus of $X_{i}$ and a negative integer $\left(-n_{i}\right)$ corresponding to the euler class of the normal bundle of $X_{i}$ in $\tilde{V}$ or equivalently the algebraic intersection number $X_{i} \cdot X_{i}$. The graph $\Gamma$ contains all topological information about the resolution, so when we are given a singularity finding a resolution will mean finding a weighted graph $\Gamma$. If $V$ is invariant under the action of a group $G$, we call $(\tilde{V}, \pi)$ an equivariant resolution if $\tilde{V}$ admits a $G$-action and $\pi$ is an equivariant map. 


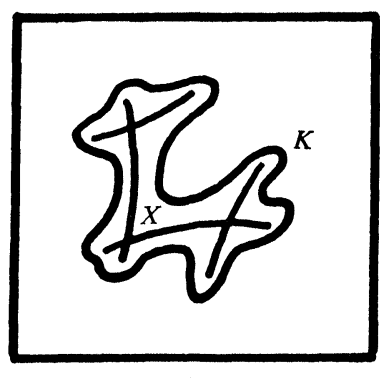

$\widetilde{V}$

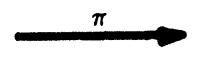

FIGURE 4

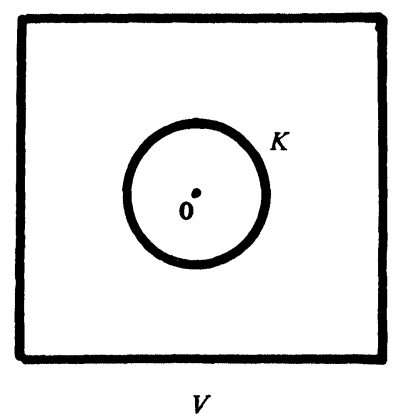

Now suppose $V$ admits a "good" $\mathbf{C}^{*}$-action, i.e. $V$ is embedded in $\mathbf{C}^{n+1}$ and it is invariant under the $\mathbf{C}^{*}$-action $t\left(z_{0}, \ldots, z_{n}\right)=\left(t^{q_{0}} z_{0}, \ldots, t^{q_{n}} z_{n}\right)$ with $q_{i}>0$. Then $K=V \cap S^{2 n+1}$ is a closed 3-manifold invariant under the $U(1) \subset C^{*}$ action. Closed 3-manifolds with circle action have been classified by Raymond [78]. The main result of Orlik and Wagreich [61] is that the $S^{1}$-manifold $K$ determines the resolution of the singularity of $V$, i.e. there is an algorithm for determining the graph $\Gamma$ from the equivariant invariants of $K=\left\{b ; g ;\left(\alpha_{1}, \beta_{1}\right), \ldots,\left(\alpha_{r}, \beta_{r}\right)\right\}$. Here $g$ is the genus of the 2-manifold $K / S^{1}$; the pair $\left\{\alpha_{i}, \beta_{i}\right\}$ refers to an orbit with isotropy $Z_{\alpha_{i}}$ and slice representation determined by $\beta_{i}, 0 \leqslant \beta_{i}<\alpha_{i}$ and $\left(\alpha_{i}, \beta_{i}\right)=1$ and $b \in Z$ is a "chern class".

Using orientations as in Orlik and Wagreich [63] we have:

THEOREM. If $V$ is an algebraic surface with a good $\mathbf{C}^{*}$-action and an isolated

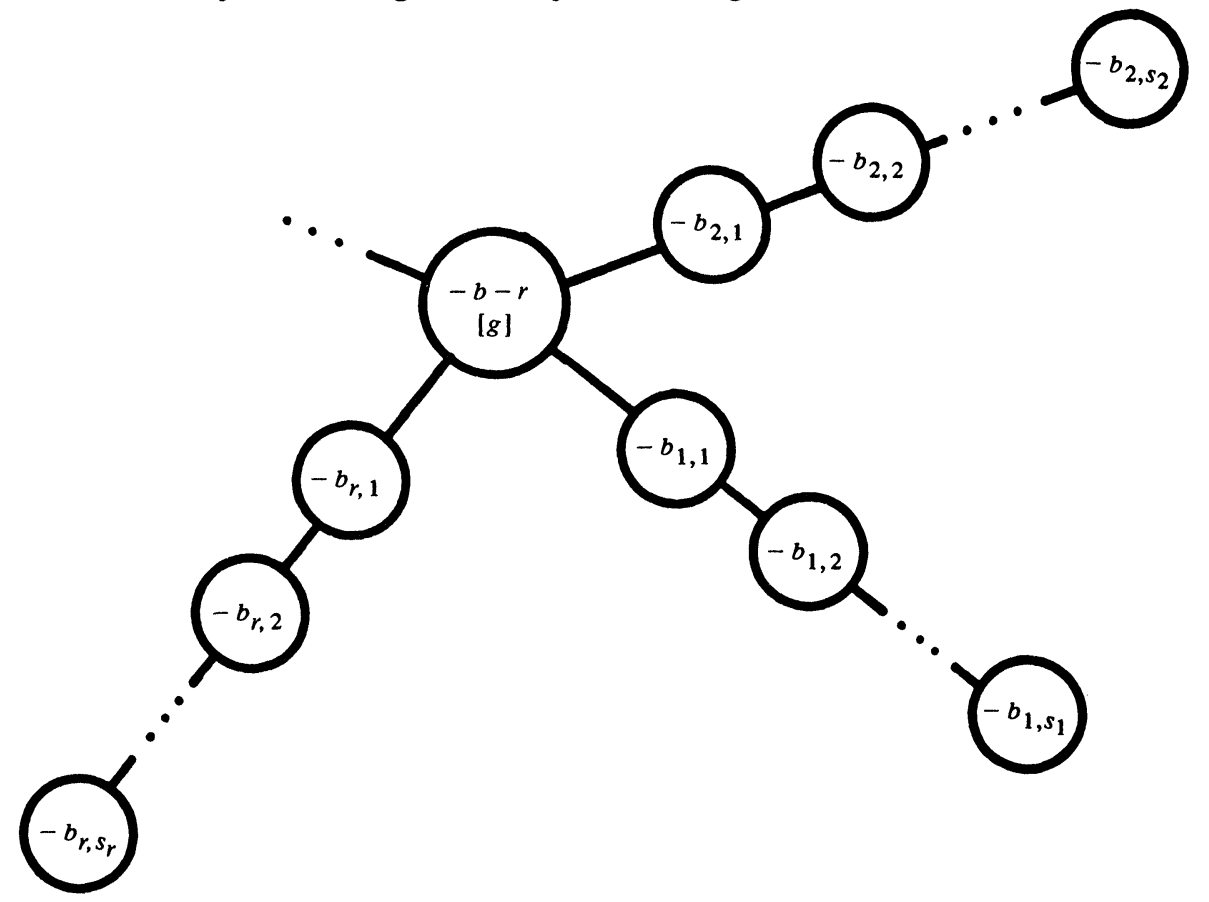

FiguRE 5 
singularity at 0 whose link is $K=\left\{b ; g ;\left(\alpha_{1}, \beta_{1}\right),\left(\alpha_{2}, \beta_{2}\right), \ldots,\left(\alpha_{r}, \beta_{r}\right)\right\}$ then the graph $\Gamma$ of an equivariant resolution is given by Figure 5, where the genus of the center curve is $\mathrm{g}$ and all other curves have genus 0 (omitted from the notation), and the integers $b_{i, j} \geqslant 2$ are obtained from the continued fraction expansions:

$$
\frac{\alpha_{i}}{\alpha_{i}-\beta_{i}}=b_{i, 1}-\frac{1}{b_{i, 2}-\frac{1}{\ldots}}
$$

If $V \subset \mathbf{C}^{3}$, then there are simple formulas for all the invariants of $K$ in terms of $d ; q_{0}, q_{1}, q_{2}$, see Orlik and Wagreich [63]. For example if we have a Brieskorn polynomial $z_{0}^{a_{0}}+z_{1}^{a_{1}}+z_{2}^{a_{2}}$, then with the notation $[a, b]=$ $\operatorname{LCM}\{a, b\}$ and $(a, b)=\operatorname{GCD}\{a, b\}$ we have:

$$
\begin{aligned}
2 g= & \frac{d^{2}}{q_{0} q_{1} q_{2}}-\frac{d\left(q_{0}, q_{1}\right)}{q_{0} q_{1}}-\frac{d\left(q_{1}, q_{2}\right)}{q_{1} q_{2}}-\frac{d\left(q_{2}, q_{0}\right)}{q_{2} q_{0}} \\
& +\frac{\left(d, q_{0}\right)}{q_{0}}+\frac{\left(d, q_{1}\right)}{q_{1}}+\frac{\left(d, q_{2}\right)}{q_{2}}-1 .
\end{aligned}
$$

There are $d /\left[q_{1}, q_{2}\right]$ orbits with $\alpha_{0}=\left(q_{1}, q_{2}\right)$ and $q_{0} \beta_{0} \equiv 1 \bmod \alpha_{0}, 0 \leqslant \beta_{0}<$ $\alpha_{0}$. Cyclic permutation of the indices determines the other exceptional orbits. Finally,

$$
b=\frac{d}{q_{0} q_{1} q_{2}}-\sum_{i=1}^{r} \frac{\beta_{i}}{\alpha_{i}} .
$$

As an illustration consider Klein's polynomial $z_{0}^{5}+z_{1}^{3}+z_{2}^{2}=0$, invariant under the $\mathrm{C}^{*}$-action with $d=30, q_{0}=6, q_{1}=10, q_{2}=15$. Direct computation yields $K=\{-1 ; 0 ;(5,1),(3,1),(2,1)\}$. Since

$$
\frac{2}{1}=2, \quad \frac{3}{2}=2-\frac{1}{2} \quad \text { and } \quad \frac{5}{4}=2-\frac{1}{2-\frac{1}{2-\frac{1}{2}}}
$$

we obtain the resolution shown in Figure 6.

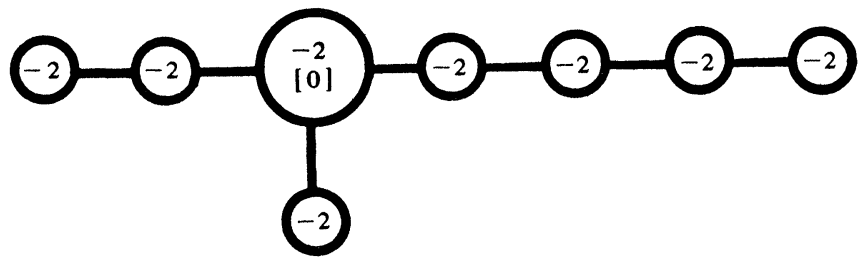

FIGURE 6

We conclude that for the binary icosahedral group $G$ the quotient $C^{2} / G$ may be embedded in $C^{3}$ as the hypersurface defined by $z_{0}^{5}+z_{1}^{3}+z_{2}^{2}=0$ and the resolution of its singularity at $\mathbf{O}$ yields the Dynkin diagram of the 
exceptional Lie group of type $E_{8}$. The table below displays this connection for all the finite subgroups of SU(2). See also Arnold [4], Brieskorn [10], Durfee [23] and Hazewinkel, Hesselink, Siersma and Veldkamp [38].

\begin{tabular}{c|c|c} 
subgroup of $\mathrm{SU}(2)$ & equation of quotient & diagram \\
\hline cyclic of order $(n+1)$ & $z_{0}^{n+1}+z_{1}^{2}+z_{2}^{2}$ & $A_{n}$ \\
binary dihedral of & $z_{0}^{2} z_{1}+z_{1}^{n-1}+z_{2}^{2}$ & $D_{n}$ \\
order $4(n-2), n \geqslant 4$ & & \\
binary tetrahedral & $z_{0}^{4}+z_{1}^{3}+z_{2}^{2}$ & $E_{6}$ \\
binary octahedral & $z_{0}^{3}+z_{0} z_{1}^{3}+z_{2}^{2}$ & $E_{7}$ \\
binary icosahedral & $z_{0}^{5}+z_{1}^{3}+z_{2}^{2}$ & $E_{8}$
\end{tabular}

8. Deformation. Let $f: \mathbf{C}^{n+1} \rightarrow \mathbf{C}$ be the germ of a holomorphic map at $\mathbf{0}$ and assume that $\boldsymbol{O}$ is an isolated critical point. Let $\theta$ be the ring of holomorphic functions at 0 and let $(\partial f)=\left(\partial f / \partial z_{0}, \ldots, \partial f / \partial z_{n}\right)$ be the ideal generated by the partial derivatives of $f$. Then $\theta /(\partial f)$ is a finite-dimensional vector space over C. In fact Palamodov [65] showed that $\operatorname{dim}_{\mathbf{C}} \theta /(\partial f)=\mu$, the Milnor number. A topology may be defined on $\theta$ so that for polynomials it is the usual topology given by the euclidean distance of the coefficients of the respective monomials. Let $\mathfrak{N}$ be the maximal ideal of holomorphic functions vanishing at 0 and let $H$ be the group of biholomorphic coordinate transformations fixing 0 . A germ $f$ is called simple if there is an open neighborhood of $f$ in $\mathfrak{N}$ which intersects only finitely many orbits of $\mathrm{H}$. Arnold [3] proved the following:

THEOREM. The simple map germs are

$$
\begin{array}{r}
A_{n}: z_{0}^{n+1}+z_{1}^{2}+z_{2}^{2}+\cdots+z_{k}^{2}, \\
D_{n}: z_{0}^{2} z_{1}+z_{1}^{n-1}+z_{2}^{2}+\cdots+z_{k}^{2}, \\
E_{6}: z_{0}^{4}+z_{1}^{3}+z_{2}^{2}+\cdots+z_{k}^{2}, \\
E_{7}: z_{0}^{3}+z_{0} z_{1}^{3}+z_{2}^{2}+\cdots+z_{k}^{2}, \\
E_{8}: z_{0}^{5}+z_{1}^{3}+z_{2}^{2}+\cdots+z_{k}^{2},
\end{array}
$$

A deformation (unfolding) of $f$ is a holomorphic map germ $F: \mathbf{C}^{n+1} \times \mathbf{C}^{k}$ $\rightarrow \mathbf{C} \times \mathbf{C}^{k}$ such that $\boldsymbol{F}(z, 0)=(f(z), 0)$. Let $t_{1}, \ldots, t_{k}$ be the coordinate functions in $\mathbf{C}^{k}$. If $f$ has an isolated critical point at $\mathbf{0}$, then the particular deformation defined by $F(z, t)=(\hat{F}(z, t), t)$ with $\hat{F}(z, t)=f(z)+\Sigma_{i=1}^{k}$ $g_{i}(z) t_{i}$, where $1, g_{1}, \ldots, g_{k}$ represent a basis of $\theta /(\partial f)$ is called semiuniversal.

The remarkable connection between the quotient spaces of finite subgroups of SU(2) and certain Lie groups was explained by Brieskorn [10]. Let $G$ be a Lie group of rank $r$ of type $A, D$ or $E$. Let $h: G \rightarrow T / W$ be the orbit map of the adjoint action sending each $x \in G$ to its conjugacy class. Here $T$ is a maximal torus and $W$ is the Weyl group of $G$. An element $x \in G$ is called regular if the dimension of its centralizer $Z_{G}(x)$ is minimal, $\operatorname{dim} Z_{G}(x)=r$. It is known that $h$ is smooth at $x$ if and only if $x$ is regular. Call $x \in G$ subregular if $\operatorname{dim} Z_{G}(x)=r+2$. Let $\mathcal{S}$ be the Lie algebra of $G, \mathcal{H}$ the Cartan subalgebra and $\chi: \mathbb{S} \rightarrow \mathcal{H C} / W$ the map induced by $h$. Let $N(\mathbb{S})$ be the 
subvariety of nilpotent elements of $\mathbb{S}$. Let $S \subset \mathbb{S}$ be a transversal slice to the subregular orbit. Then $S$ has codimension 2 in $N(\mathbb{S})$ and Brieskorn [10] shows:

THEOREM. The singularity of the surface $S \cap N(\mathbb{S})$ corresponds to the type of the Lie group $G$ and the restriction $\left.\chi\right|_{S}: S \rightarrow \mathcal{H} / W$ realizes a semiuniversal deformation of this singularity.

This construction was extended to the Lie algebras of type $B_{n}, C_{n}, F_{4}$ and $G_{2}$ by Slodowy [85].

9. Monodromy. If $f: \mathbf{C}^{n+1} \rightarrow \mathbf{C}$ has an isolated critical point at $\mathbf{O}$ and $F$ : $\mathbf{C}^{n+k+1} \rightarrow \mathbf{C}^{k+1}$ is a semiuniversal deformation of $f$, then the set of critical values of $F$ forms a hypersurface $D \subset C^{k+1}$ which is called the discriminant. For a suitable open set $S \subset C^{k+1}$ and for $S^{\prime}=S-D$, the fibers of $F$ over $S^{\prime}$ are smooth with homotopy type a bouquet of $n$-spheres. Let $X_{s}$ be a typical fiber, $s \in S^{\prime}$. The image of the natural map $\pi_{1}\left(S^{\prime}, s\right) \rightarrow \operatorname{Aut}\left(H^{n}\left(X_{s} ; \mathbf{Z}\right)\right)$ is called the monodromy group $M$. For more information on this group and its connection with the intersection form on $X_{s}$ see Lamotke [47]. The surjection $\pi_{1}\left(S^{\prime}, s\right) \rightarrow M$ gives rise to a regular covering $\tilde{S}^{\prime} \rightarrow S^{\prime}$ with $M$ as group of covering transformations. It can be shown that $M$ is generated by reflections, and that it is finite if and only if $X_{0}$ is a simple hypersurface singularity of even dimension. In general if $G$ is a finite reflection group acting on $V=C^{l}$ then $V / G$ is naturally isomorphic to $\mathbf{C}^{l}$, so in case of simple singularities the same holds for $H^{n}\left(X_{s} ; C\right) / M$. Brieskorn [10] proved that in this case the covering $\tilde{S}^{\prime} \rightarrow S^{\prime}$ extends to a covering $\tilde{S} \rightarrow S$ ramified over $D$ such that (for suitable $F)$ the resulting map is equivalent to the orbit map $H^{n}\left(X_{s} ; \mathrm{C}\right) \rightarrow$ $H^{n}\left(X_{s} ; C\right) / M$. Looijenga [50] extended these results to simple-elliptic singularities, making explicit use of the fact that if $X_{0}$ admits a $C^{*}$-action, then the whole construction may be made $C^{*}$-equivariant, see also Pinkham [68], [69].

10. Newton diagram. Let $\mathbf{N}$ be the set of nonnegative integers and let $\mathbf{R}_{+}$be the set of nonnegative real numbers. Let $K$ be a subset of $\mathrm{N}^{n+1}$. Denote by $\Gamma_{+}(K)$ the convex hull of $\cup_{m \in K}\left(m+\mathbf{R}_{+}^{n+1}\right)$ in $\mathbf{R}_{+}^{n+1}$. The union of all compact faces of $\Gamma_{+}(K)$ is the Newton diagram $\Gamma(K)$ of $K$. For a power series in $(n+1)$ variables $z=\left(z_{0}, \ldots, z_{n}\right), f=\Sigma_{m \in N^{n+1}} a_{m} z^{m}, a_{m} \in \mathbf{C}$ we define the support of $f, \operatorname{supp} f=\left\{m \in \mathbf{N}^{n+1} \mid a_{m} \neq 0\right\}$. Let $\Gamma(f)=\Gamma(\operatorname{supp} f)$ be the Newton diagram of $f$. Let $\Gamma_{-}(f)$ be the cone over $\Gamma(f)$ with cone point the origin. Let $\gamma$ be a closed face of $\Gamma(f)$ and define $f_{\gamma}=\Sigma_{m \in \gamma} a_{m} z^{m}$. The main part of $f$ is the polynomial $\Sigma_{m \in \Gamma(f)} a_{m} z^{m}$. The main part of $f$ is called nondegenerate if for any closed face $\gamma \in \Gamma(f)$ the polynomials $z_{0}\left(\partial f_{\gamma} / \partial z_{0}\right), \ldots, z_{n}\left(\partial f_{\gamma} / \partial z_{n}\right)$ have no common zero in $\left\{z \in \mathbf{C}^{n+1} \mid z_{0} \ldots z_{n} \neq\right.$ $0\}$. Kushnirenko [46] proved the following interesting connection between the Newton diagram and the Milnor number.

THEOREM. If $f$ has an isolated critucal point at the origin and if the main part of $f$ is nondegenerate, then

$$
\mu=(n+1) ! V_{n+1}-n ! V_{n}+\cdots+(-1)^{n} V_{1}+(-1)^{n+1}
$$

where $V_{n+1}$ is the $(n+1)$-dimensional volume of $\Gamma_{-}(f)$ and for $1 \leqslant q \leqslant n V_{q}$ 
is the sum of the q-dimensional volumes of the intersections of $\Gamma_{-}(f)$ with the $q$-dimensional coordinate planes.

For isolated singularities the Milnor fiber has homology only in dimensions 0 and $n$, the latter of rank $\mu$. Thus its euler characteristic equals $\chi=1+$ $(-1)^{n} \mu$ so

$$
\chi=V_{1}-2 ! V_{2}+\cdots+(-1)^{n}(n+1) ! V_{n+1} .
$$

Varchenko [88] has shown that this expression gives the euler characteristic of the Milnor fiber regardless of the singularity of $f$, provided its main part is nondegenerate. The Newton diagram of a quasihomogeneous polynomial lies in a hyperplane so it is particularly tractable. This enables Oka [57] to obtain topological conclusions from assumptions about the behavior of the Newton diagram.

11. Automorphic forms. As we noted in the introduction, Klein's variety defined by $z_{0}^{5}+z_{1}^{3}+z_{2}^{2}=0$ in $\mathbf{C}^{3}$ is the quotient space of $\mathbf{C}^{2}$ by the action of the binary icosahedral group $G$. The corresponding 3-manifold $K$ whose orbit invariants were obtained in $\$ 7$ is the homogeneous space $\mathrm{SU}(2) / G$. The natural question arises, which singularities are obtained as quotients by a group action. For finite subgroups of SU(2) the answer was given by Klein and the corresponding singularities have turned up in a variety of different contexts. For the general case see Milnor [52] and Raymond and Vasquez [79]. The next construction is also a generalization of classical results.

Suppose $G \subset \mathrm{GL}(2 ; \mathbf{C})$ is acting properly discontinuously in some domain of holomorphy $D \subset \mathbf{C}^{2}$. Let $\bar{G} \subset \operatorname{PSL}(2 ; \mathbf{C})$ be its image acting on $\mathbf{C P}^{1}=S^{2}$. If $G$ is finite, then $\bar{G}$ is properly discontinuous on all of $\mathbf{C P}^{1}$. Otherwise $\bar{G}$ is properly discontinuous on the euclidean plane $P_{E}=\mathrm{C}$ or the hyperbolic plane $P_{H}=\{z \in \mathbf{C}|| z \mid<1\}$. In the latter cases the domain $D$ is a trivial $\mathbf{C}^{*}$ bundle over $P_{E}$ or $P_{H}$ and it is not hard to compute the action of $G$ with respect to a trivialization.

More generally if $V$ is a complex variety and $G$ a group of its automorphisms, we call a line bundle $p: L \rightarrow V$ with $G$-action an automorphic factor with respect to $G$ if

(i) $p(g x)=g(p(x))$ for all $x \in L, g \in G$,

(ii) $g: L \rightarrow L$ by $x \rightarrow g x$ restricted to $p^{-1}(z)$ defines a linear map $p^{-1}(z) \rightarrow p^{-1}(g z)$ for all $z \in V, g \in G$.

In our case $L=V \times \mathrm{C}$, with $V=P_{E}$ or $P_{H}$ corresponding to the insertion of a zero section to the bundle $V \times \mathbf{C}^{*}$. Here the structure of the automorphic factor on $L$ is determined by a function $h: G \times V \rightarrow \mathrm{C}^{*}$, holomorphic in $z$ and subject to the condition

$$
h\left(g g^{\prime}, z\right)=h\left(g, g^{\prime} z\right) h\left(g^{\prime}, z\right) \text { for all } g, g^{\prime} \in G, z \in V .
$$

The action on $L$ is given by $g(z, a)=(g z, h(g, z) a)$. If $L$ and $L^{\prime}$ are automorphic factors for $G$, then $L \otimes L^{\prime}$ is a line bundle with $G$-action $g\left(1 \otimes 1^{\prime}\right)=g(1) \otimes g\left(1^{\prime}\right)$ for all $z \in V, \quad 1 \in L_{z}, 1^{\prime} \in L_{z}^{\prime}$. Let $L^{\otimes m}=L$ $\otimes \cdots \otimes L(m$ times $)$ and $L^{\otimes 0}=V \times C$ with $g(z, a)=(g z, a)$. Similarly we can define dual automorphic factors. 
In the case of finite groups $G$ the algebra of $G$-invariant homogeneous polynomials is finitely generated and there is a natural isomorphism between the variety defined by it and $C^{2} / G$. If $G$ is infinite, then the only $G$-invariant polynomials are constants. The replacement is a G-automorphic form on $V$ relative to the automorphic factor $L:$ a holomorphic function $f: L \rightarrow \mathrm{C}$ such that

(i) $f(g x)=f(x)$ for all $x \in L, g \in G$,

(ii) the restriction of $f$ to each fiber $L_{z}$ is a homogeneous linear form.

The $G$-automorphic forms on $V$ relative to $L$ form a $C$-vector space $A(G$; $L)$, and if $f \in A(G ; L), f^{\prime} \in A\left(G ; L^{\prime}\right)$ then $f \cdot f^{\prime} \in A\left(G ; L \otimes L^{\prime}\right)$. Thus

$$
A_{L}(G)=\bigoplus_{m=0}^{\infty} A\left(G ; L^{\otimes m}\right)
$$

is a graded subalgebra of the algebra of holomorphic functions on $L$ consisting of $G$-invariant functions on $L$ whose restrictions are polynomials in each fiber, called the algebra of $G$-automorphic forms on $V$ relative to $L$. The elements of $A_{L}(G)_{m}=A\left(G ; L^{\otimes m}\right)$ are called automorphic forms of weight $m$.

This algebra is a classical object, see Fricke and Klein [28]. Its connection with our present subject, the isolated singularities of quasihomogeneous polynomials, was known to F. Klein and H. Poincaré. The recent advances are due to Conner and Raymond [16], Raymond and Vasquez [79], Milnor [52], Dolgachev [19], [20], Neumann [55], Wagreich [89], [90] Gorbatsevich [33].

The triple $(V, G, L)$ is called admissible if

(i) there is a normal subgroup $G^{\prime} \subset G$ which acts freely and discretely on $V$,

(ii) the factor space $V / G$ is a compact analytic space,

(iii) for some $G^{\prime} \subset G$ satisfying (i), the quotient $L / G^{\prime}$ determines a positive line bundle over the manifold $V / G^{\prime}$.

As usual given a ring $A$ the space $\operatorname{Spec}(A)$ is its spectrum of maximal ideals. The following result of Dolgachev was announced in [19] and proved in [20].

Theorem. Let $(V, G, L)$ be an admissible triple. Then the affine algebraic variety $\operatorname{Spec} A(L)$ is a quasihomogeneous surface with isolated singularity at $x_{0}$ defined by the maximal ideal $A(L)_{+}=\bigoplus_{i>0} A(L)_{i}$. Conversely, each quasihomogeneous surface with good $\mathbf{C}^{*}$-action and isolated singularity is isomorphic to the variety Spec $A(L)$ for some admissible triple $(V, G, L)$. Moreover, we may assume that $G$ acts freely outside the zero section of $L$.

The question of which singularity is obtained for a given $G \subset \mathrm{GL}(2 ; \mathrm{C})$ is currently investigated by Orlik and Wagreich [64].

12. Complements of hyperplanes. Let $V=\mathrm{C}^{l}$ and let $A_{1}, \ldots, A_{n}$ be hyperplanes through the origin. Let $M=V-\cup_{i=1}^{n} A_{i}$. If $\varphi_{1}, \ldots, \varphi_{n}$ are linear forms such that $\operatorname{ker} \varphi_{i}=A_{i}$, then in general the polynomial $f=\varphi_{1} \cdots \varphi_{n}$ has a nonisolated singularity at 0 . Note that $M$ is the total space of the Milnor fibration. Computation of $H^{*}(M)$ first appeared in the work of Arnold [2] and Brieskorn [11] in connection with finite (real) reflection groups, where the $A_{i}$ are the reflecting hyperplanes and $M$ is the set of regular 
vectors. Brieskorn [11] computed in principle $H^{*}(M)$ for arbitrary $M$. In a forthcoming paper Orlik and Solomon [60] give an algorithm for computing $H^{*}(M)$ as follows. Assume complex coefficients throughout. Introduce a partial order in the set $L$ of all intersections of the $A_{i}$ by reverse inclusion, i.e. $X<X^{\prime}$ if $X \supseteq X^{\prime}$. Define the Möbius function of $L$ inductively as follows: $\mu(V)=1$ and for $X \in L, X \neq V: \Sigma_{V \leqslant X^{\prime} \leqslant X} \mu\left(X^{\prime}\right)=0$. The partially ordered set $L$ turns out to be a geometric lattice with minimal element $V$ and maximal element $\cap_{i=1}^{n} V_{i}$. The rank function of $L$ is given by $r(X)=\operatorname{codim}_{\mathbf{C}} X$ and the atoms of $L$ are the $A_{i}$.

THEOREM. The Poincaré polynomial of $M$ is given by

$$
P(M ; t)=\sum_{X \in L} \mu(X)(-t)^{r(X)} .
$$

If $G \subset \mathrm{GL}(V)$ is a finite group which operates on the set of hyperplanes $A_{1}, \ldots, A_{n}$ by permutation, then $G$ has a representation on $H^{*}(M)$. The trace is obtained as follows. Let $g \in G$ and let $L^{g}=\{X \in L \mid g X<X\}$. Then $L^{g}$ is a poset (but not necessarily a geometric lattice), with its Möbius function $\mu_{g}$.

THEOREM. The trace of $g \in G$ on $H^{*}(M)$ is given by

$$
\sum_{p>0} \operatorname{tr}\left(g \mid H^{p}(M)\right) t^{p}=\sum_{X \in L^{g}} \mu_{g}(X)(-t)^{r(X)}
$$

where $r(X)=\operatorname{codim}_{\mathbf{C}} X$ is the rank function of $L$.

If $G$ is an irreducible finite real reflection group and $A_{i}$ are its complexified reflecting hyperplanes, then Brieskorn [11] proved that $P(M ; t)=\Pi_{i=1}^{l}(1+$ $\left.m_{i} t\right)$ where $m_{1}, \ldots, m_{l}$ are the exponents of $G$.

THEOREM. If $G$ is an irreducible finite unitary reflection group and $A_{1}, \ldots, A_{n}$ are its reflecting hyperplanes, then

$$
P(M ; t)=\prod_{i=1}^{l}\left(1+n_{i} t\right)
$$

where the $n_{1}, \ldots, n_{l}$ are positive integers. If $G$ is real, then $n_{i}=m_{i}$.

Deligne [17] showed that $M$ is a $K(\pi, 1)$ space if $G$ is a real reflection group. It is not known whether the same holds for unitary reflection groups.

13. Related topics. The subject of Singularities has grown tremendously in the last decade. There is no adequate survey of the subject as a whole. The material for this article was selected on the basis of its connection with Transformation Groups. For the convenience of the reader interested in other aspects we shall list references for some major areas of current activity.

(i) Golubitsky and Guillemin's book [31] is an excellent introduction to the Thom-Mather theory of the stability of mappings and the stability of unfoldings, the classification of singularities, the Thom-Boardman stratification and catastrophe theory. For other treatments see Wassermann [91] and Gibson, Wirthmüller, du Plessis and Looijenga [29]. Poènaru [70] has developed an equivariant theory. Two recent collection of papers [84], [86] deal with 
structural stability and related questions, see also the Liverpool symposium volumes [71], [72]. A treatment of the elementary catastrophes appears in Woodcock and Poston [92] with appealing pictures. Many applications to physics, economics and biology are discussed in a collection of papers by Zeeman [93].

(ii) Arnold [4] gives a general survey of his classification of singularities with small Milnor number, the adjacency problem and applications to caustics, wavefronts and oscillating integrals. Recent advances in the classification and adjacency are reported by Arnold [5] and by Siersma in [80].

(iii) Methods using stratifications, polar curves, Lefschetz pencils and Morse theory have been applied in various forms, see Hironaka's article in [80], A'Campo [1], Lê's papers in [73], [80], [83], Hamm and Lê [36] and Teissier's reports in [73], [83].

(iv) Many tools of algebraic geometry are used in singularities. Lipman gives an introduction to the resolution of singularities in [73]. The problems of equisingularity and deformations are treated by several authors in [73], [80], [83]. The enumerative theory is reviewed by Kleiman in [80]. Tessier treats the invariants of the discriminant in [80]. The fact that the Milnor fiber is a Stein manifold and therefore its cohomology with complex coefficients is isomorphic to the cohomology of its de Rham complex of holomorphic differential forms is used by Brieskorn [12] and Greuel [34] to develop a singular local Gauss-Manin connection, which gives an algebraic method of computing the monodromy. Steenbrink's article in [80] is a good introduction to mixed Hodge structures.

(v) The actions of the complex torus are treated by Kempf, Knudsen, Mumford and Saint-Donat [44]. Connections between $\mathbf{C}^{*}$-actions and vector fields on compact Kaehler manifolds are discussed by Carrell and Lieberman [13] and by Carrell and Sommese [14].

\section{REFERENCES}

1. N. A'Campo, Le groupe de monodromie du déploiement des singularités isolées de courbes planes, Math. Ann. 213 (1975), 1-32.

2. V. I. Arnold, The cohomology of the ring of the colored braid group, Math. Notes Acad. Sci. USSR 5 (1969), 138-140.

3. __ Normal forms for functions near degenerate critical points, the Weyl groups $A_{k}, D_{k}$, $E_{k}$ and Lagrangian singularities, Functional Anal. Appl. 6 (1972), 254-272.

4. __ Critical points of smooth functions, Proc. Internat. Congr. Math. 1 (1974), 19-39.

5. __ Local normal forms of functions, Invent. Math. 35 (1976), 87-109.

6. M. F. Atiyah and R. Bott, A Lefschetz fixed point formula for elliptic complexes: II. Applications, Ann. of Math. (2) 88 (1968), 451-491.

7. G. E. Bredon, Examples of differentiable group actions, Topology 3 (1965), 115-122.

8. Regular $O(n)$-manifolds, suspension of knots, and knot periodicity, Bull. Amer. Math. Soc. 79 (1973), 87-91. $1-14$.

9. E. Brieskorn, Beispiele zur Differentialtopologie von Singularitäten, Invent. Math. 2 (1966),

10. __ Singular elements of semi-simple algebraic groups, Actes, Congrés Internat. Math. 2 (1970), 279-284.

11. __ Sur les groupes de tresses, Sém. Bourbaki 401 (1971).

12. $\longrightarrow$ Die Monodromie der isolierten Singularitäten von Hyperflächen, Manuscripta Math. 2 (1970), 103-161. 
13. J. Carrell and D. Lieberman, Holomorphic vector fields and Kaehler manifolds, Invent. Math. 21 (1973), 303-309.

14. J. Carrell and A. Sommese, Some topological aspects of $\mathbf{C}^{*}$-actions on compact Kaehler manifolds (preprint).

15. __,$S L(2 ; \mathrm{C})$ actions on compact Kaehler manifolds 1 (preprint).

16. P. Conner and F. Raymond, Holomorphic Seifert fibering, Lecture Notes in Math., vol. 299, Springer-Verlag, Berlin and New York, 1972, pp. 124-204.

17. P. Deligne, Les immeubles des groupes de tresses, Invent. Math. 17 (1972), 273-302.

18. J. A. Dieudonné and J. B. Carrell, Invariant theory, old and new, Academic Press, New York, 1971.

19. I. V. Dolgachev, Automorphic forms and quasihomogeneous singularities, Functional Anal. Appl. 9 (1975), 149-151.

20. __ Automorphic forms and weighted homogeneous polynomials (preprint).

21. Weighted projective varieties (preprint).

22. A. H. Durfee, Foliations of odd-dimensional spheres, Ann. of Math. (2) 96 (1972), 407-411.

23. Fifteen characterizations of rational double points and simple critical points,

L'Enseignement Math. (to appear).

24. A. H. Durfee and L. H. Kauffman, Periodicity of branched cyclic covers, Ann. of Math. (2) 218 (1975), 157-174.

25. G. Edmunds, On the orbit space of a weighted homogeneous hypersurface, Proc. London Math. Soc. 29 (1974), 684-698.

26. L. P. Eisenhart, Riemannian geometry, Princeton Univ. Press, Princeton, N. J., 1926.

27. D. Erle, Die quadratische Form eines Knotens und ein Satz über Knotenmannigfaltigkeiten, J.

Reine Angew. Math. 236 (1969), 174-218.

28. R. Fricke and F. Klein, Vorlesungen über die Theorie der Automorphen Functionen, Leipzig, Teubner 1897, 1901, 1912.

29. C. G. Gibson, K. Wirthmüller, A. A. du Plessis and E. J. N. Looijenga, Topological stability of smooth mappings, Lecture Notes in Math., vol. 552, Springer-Verlag, Berlin and New York, 1976.

30. C. H. Giffen, Smooth homotopy projective spaces, Bull. Amer. Math. Soc. 75 (1969), 509-513.

31. M. Golubitsky and V. Guillemin, Stable mappings and their singularities, Springer-Verlag, Berlin and New York, 1973.

32. M. Golubitsky and D. Schaeffer, $A$ theory for imperfect bifurcation via singularity theory, MRC Preprint (1978).

33. V. V. Gorbatsevich, Three-dimensional homogeneous spaces, Sibirsk Mat. Ż. 18 (1977), 280-293.

34. G.-M. Greuel, Der Gauss-Manin-Zusammenhang isolierter Singularitäten von vollständigen Durchschnitten, Math. Ann. 214 (1975), 235-266.

35. H. A. Hamm, Lokale topologische Eigenschaften komplexer Räume, Math. Ann. 191 (1971), 235-252.

36. H. A. Hamm and Lê Dũng Tráng, Un théorème de Zariski du type de Lefschetz, Ann. Sci. École Norm. Sup. (4) 6 (1973), 317-355.

37. H. A. Hamm and G.-M. Greuel, Invarianten quasihomogener vollständiger Durchschnitten, Invent. Math. 49 (1978), 67-86.

38. M. Hazewinkel, W. Hesselink, D. Siersma and F. D. Veldkamp, The ubiquity of CoxeterDynkin diagrams, Nieuw Arch. Wisk. 25 (1977), 257-307.

39. M. W. Hirsch and J. Milnor, Some curious involutions of spheres, Bull. Amer. Math. Soc. 70 (1964), 372-377.

40. F. Hirzebruch, Singularities and exotic spheres, Sém. Bourbaki 314 (1966).

41. W. Y. Hsiang, A survey of regularity theorems in differentiable compact transformation groups, Proc. Conf. on Transformation Groups, Springer-Verlag, Berlin and New York, 1968, 77-124.

42. K. Jänich, Differenzierbare Mannigfaltigkeiten mit Rand als Orbiträume differenzierbarer G-Mannigfaltigkeiten ohne Rand, Topology 5 (1966), 301-320.

43. L. H. Kauffman and W. D. Neumann, Products of knots, branched fibrations and sums of singularities, Topology 16 (1977), 369-393. 
44. G. Kempf, F. Knudsen, D. Mumford and B. Saint-Donat, Toroidal embeddings. I, Lecture Notes in Math., vol. 339, Springer-Verlag, Berlin and New York, 1973.

45. F. Klein, Lectures on the Icosahedron and the Solution of Equations of the Fifth Degree, Dover 1956. (Original: Teubner, Leipzig, 1884).

46. A. G. Kushnirenko, Polyèdres de Newton et nombres de Milnor, Invent. Math. 32 (1976), 1-31.

47. K. Lamotke, Die Homologie isolierter Singularitäten, Math. Z. 143 (1975), 27-44.

48. H. B. Lawson, Jr., Codimension-one foliations of spheres, Ann. of Math. 94 (1971), 494-503.

49. S. Lie and F. Engel, Theorie der Transformationsgruppen. III, (Leipzig: Teubner 1893); reprinted New York: Chelsea 1970.

50. E. Looijenga, On the semi-universal deformation of a simple-elliptic hypersurface singularity,

Part II: the discriminant, Topology 17 (1978), 23-40.

51. J. Milnor, Singular points of complex hypersurfaces, Annals of Math. Studies, No. 61, Princeton Univ. Press, Princeton, N. J., 1968.

52. , On the 3-dimensional Brieskorn manifold $M(p, q, r)$, Annals of Math. Studies no.

84, Princeton Univ. Press, Princeton, N. J., 1975, 175-225.

53. J. Milnor and P. Orlik, Isolated singularities defined by weighted homogeneous polynomials, Topology 9 (1970), 285-293.

54. W. D. Neumann, $S^{1}$-actions and the $\alpha$-invariant of their involutions, Bonn Math. Schr. 14 (1970).

55. 285-293. Brieskorn complete intersections and automorphic forms, Invent. Math. 42 (1977),

56. M. Oka, On the homotopy types of hypersurfaces defined by weighted homogeneous polynomials, Topology 12 (1973), 19-32.

57. , On the topology of the Newton boundary. II (generic weighted homogeneous singularity) (preprint).

58. P. Orlik and R. Randell, The monodromy of weighted homogeneous singularities, Invent. Math. 39 (1977), 199-211.

59. P. Orlik and L. Solomon, Singularities. II; Automorphisms of forms, Math. Ann. 231 (1978), 229-240.

60. Combinatorics and topology of complements of hyperplanes (preprint).

61. P. Orlik and P. Wagreich, Isolated singularities of algebraic surfaces with $\mathbf{C}^{*}$-action, Ann. of Math. (2) 93 (1971), 205-228.

62. _ Singularities of algebraic surfaces with $C^{*}$-action, Math. Ann. 193 (1971), 121-135.

63. ___ Algebraic surfaces with $k^{*}$-action, Acta Math. 138 (1977), 45-81.

64. (in preparation).

65. V. P. Palamodov, Multiplicity of holomorphic mappings, Functional Anal. Appl. 1 (1967), 218-266.

66. T. Petrie, Free metacyclic group actions on homotopy spheres, Ann. of Math. (2) 94 (1971), $108-124$.

67. F. Pham, Introduction a l'étude topologique des singularitiés de Landau, Memorial des Sciences Math. 164, Paris, 1967.

68. H. Pinkham, Normal surface singularities with $C^{*}$-action, Math. Ann. 227 (1977), 183-193.

69. __ Deformations of normal surface singularities with $\mathbf{C}^{*}$-action (preprint).

70. V. Poènaru, Singularités $C^{\infty}$ en présence de symétrie, Lecture Notes in Math., vol. 510, Springer-Verlag, Berlin and New York, 1976.

71. Proceedings of Liverpool Singularities-Symposium I, Lecture Notes in Math., vol. 192, Springer-Verlag, Berlin and New York, 1971.

72. Proceedings of Liverpool Singularities-Symposium II, Lecture Notes in Math., vol. 209, Springer-Verlag, Berlin and New York, 1971.

73. Algebraic geometry-Arcata 1974, edited by R. Hartshorne, Proc. Sympos. Pure Math., vol. 29, American Math. Soc., Providence, R. I., 1975.

74. R. Randell, The homology of generalized Brieskorn manifolds, Topology 14 (1975), 347-355.

75. Index invariants of orbit spaces, Math. Scand. 36 (1975), 263-270.

76. __ Quotients of complete intersections by $\mathbf{C}^{*}$-actions (preprint).

77. __ The Milnor number of isolated complete intersection singularities with $\mathrm{C}^{*}$-action (preprint). 
78. F. Raymond, Classification of the actions of the circle on 3-manifolds, Trans. Amer. Math. Soc. 131 (1968), 51-78.

79. F. Raymond and A. Vasquez, Closed 3-manifolds whose universal covering is a Lie group (In preparation).

80. Real and complex singularities (Proc. Ninth Nordic Summer School/NAVF Sympos. Math., Oslo 1976), Sijthoff \& Noordhoff, Alphen aan den Rijn, 1977.

81. R. Schultz, Improved estimates for the degree of symmetry of certain homotopy spheres, Topology 10 (1971), 227-235.

82. M. Sebastiani and R. Thom, Un résultat sur la monodromie, Invent. Math. 13 (1971), 90-96.

83. Singularités à Cargèse, (Rencontre, 1972) Astérisque, Nos. 7 \& 8, Soc. Math. France, Paris, 1973.

84. Singularités d'applications différentiables (Séminaire, Plains-sur-Bex, 1975), Lecture Notes in Math., vol. 535, Springer-Verlag, Berlin and New York, 1976.

85. P. Slodowy, Einfache Singularitäten und einfache algebraische Gruppen, Regensburger Math. Schriften 21978.

86. Structural stability, the theory of catastrophes, and applications in the sciences (Proc. Conf. Battelle Seattle Res. Center, 1975), Lecture Notes in Math., vol. 525, Springer-Verlag, Berlin and New York, 1976.

87. W. Thurston, Existence of codimension-one foliations, Ann. of Math. (2) 104 (1976), 249-268.

88. A. N. Varchenko, Zeta-function of monodromy and Newton's diagram, Invent. Math. 37 (1976), 253-262.

89. P. Wagreich, Algebras of automatic forms with few generators (preprint).

90. __ Automorphic forms and singularities of complex surfaces (preprint).

91. G. Wassermann, Stability of unfoldings, Lecture Notes in Math., vol. 393, Springer-Verlag, Berlin and New York, 1974.

92. A. E. R. Woodcock and T. Poston, A geometrical study of the elementary catastrophes, Lecture Notes in Math., vol. 373, Springer-Verlag, Berlin and New York, 1974.

93. E. C. Zeeman, Catastrophe theory, Addison-Wesley, Reading, Mass., 1977.

Department of Mathematics, University of Wisconsin, Madison, Wisconsin 53706 